•论坛・

\title{
IPBES工作进展及我国对策建议
}

\author{
潘玉雪 张博雅 吴 杨 戴逢斌 田 瑜*
}

(中国环境科学研究院环境基准与风险评估国家重点实验室, 北京 100012)

\begin{abstract}
摘要：作为生物多样性保护领域的首个政府间机制，生物多样性和生态系统服务政府间科学政策平台(IPBES)正 在对全球生物多样性和生态系统保护政策的制定和实施产生重要影响, 其评估成果大大提升了全球决策者对生物 多样性的关注。2019年4月, IPBES在法国巴黎召开第七次全体会议, 会议总结了第一轮工作方案(2014-2019)各目 标的实现情况, 通过了第二轮工作方案(2019-2030), 为未来10年重点工作和评估方向提供指导。本文结合两轮工 作方案的具体目标, 分析研判了IPBES工作及评估进展, 对各成员国主要观点进行了梳理, 提出了中国的应对策 略。IPBES评估发现, 人类面临的生物多样性问题越来越多元化、复杂化, IPBES正通过发布一系列的评估报告, 将 生物多样性保护问题进一步主流化、政治化。我们建议，作为全球生物多样性最为丰富的国家之一，中国应该充 分认识到IPBES的作用, 积极参与有关进程, 加强跨部门跨学科协助, 促进国际合作与交流, 提升我国在生物多样 性领域相关谈判中的主动权和话语权。
\end{abstract}

关键词: 生物多样性; 生态系统服务; 第二轮工作方案(2019-2030); 生物多样性和生态系统服务平台

\section{The latest developments of IPBES and China's countermeasures}

Yuxue Pan, Boya Zhang, Yang Wu, Fengbin Dai, Yu Tian*

State Key Laboratory of Environmental Criteria and Risk Assessment, Chinese Research Academy of Environmental and Sciences, Beijing 100012

\begin{abstract}
The Intergovernmental Science-Policy Platform on Biodiversity and Ecosystem Services (IPBES) is the first intergovernmental policy mechanism for biodiversity conservation. To date, IPBES has greatly affected the development and implementation of global biodiversity and ecosystem protection policies and has significantly increasing global policymakers' concern on biodiversity. IPBES held its 7th Plenary Session in April 2019 in Paris, France. The plenary summarized the achievements of the goals in its first work programme (2014-2019), and approved the second work programme (2019-2030), which will guide the crucial work and assessments of the next decade. This paper analyzed IPBES progress and main points of IPBES members based on objectives in the two work programmes, and proposed China's countermeasures. Based on the IPBES assessments, biodiversity related issues are becoming more diversified and complex. IPBES is further mainstreaming and politicizing biodiversity conservation issues by launching a series of deliverables. As China is one of the most biodiverse countries in the world, we recommend China recognize the important role of IPBES and enhance its initiative and voice in relevant negotiations by encouraging active participation in IPBES processes, strengthening cross-sectoral and interdisciplinary collaboration, and promoting international cooperation and exchanges.
\end{abstract}

Key words: biodiversity; ecosystem services; Second Work Programme (2019-2030); IPBES

2019年4月, 在法国巴黎召开的生物多样性和 生态系统服务政府间科学政策平台(The Intergovernmental Science-Policy Platform on Biodiversity and Ecosystem Services, IPBES)第七次全体会议上,
各成员国审议通过了2019-2030年工作方案(即第二 轮工作方案), 确定了平台未来十年将以滚动的方 式开展工作。该工作方案将接替 2013年通过的 2014-2019年工作方案(第一轮工作方案) (田瑜等, 
2015)，指导IPBES未来的工作重点和评估方向。目 前IPBES第一轮工作方案有关目标已经基本达成, 其核心目标, 即生物多样性和生态系统服务全球评 估，亦于此次全体会议上获得通过并正式发布。

第二轮工作方案将科学评估2030年全球生物 多样性目标、《2030年可持续发展议程》(包括可持 续发展目标) 和《巴黎协定》的阶段进展, 促进各方 努力实现有关目标。《生物多样性公约》(CBD)第14 次缔约方大会(COP14)对IPBES第二轮工作方案的 进程及评估专题十分重视, 设立了专门的议题进行 讨论, 并提出要充分利用IPBES 全球评估报告为 2020 后全球生物多样性框架(以下简称“2020后框 架”)提供建议。

为保障第二轮工作方案的包容性、公正性和透 明性, IPBES秘书处向各成员国和利益敒关方及有 关公约就IPBES第二轮工作方案的编制步骤和内容 征集了建议，并在第七次全体会议上进行了激烈的 讨论与谈判, 最后一致通过。该工作方案的通过对 IPBES未来发展具有重要指导意义, 标志着IPBES 新一轮评估工作的开始。分析研判IPBES发展现状 及其评估进展, 了解其未来评估目标及方向, 有助 于我国调整应对策略, 更好地参与IPBES工作, 提 升话语权。本文通过总结IPBES第一轮工作方案的 实施情况, 结合第二轮工作方案的重点评估专题及 各方关注焦点, 就我国继续参与IPBES相关工作提 出建议。

\section{1 第一轮工作方案的成果及影响}

IPBES第二次全体会议通过的第一轮工作方案 (2014-2019), 不仅体现了 IPBES 的四项基本职能 (开展评估、能力建设、政策支持和创造新知识), 同 时反映了各成员国、利益做关方和多边环境协定的 关注点。

\section{1 取得的成果}

IPBES第一轮工作方案共包含了四项跨领域综 合目标(田瑜等, 2015), 并在此目标指导下确定了 IPBES在2014-2019年期间所要开展的工作，包括5 项多尺度生物多样性和生态系统服务综合评估、4 项专题评估及 2 项方法评估。截至 2019年10月, IPBES已经陆续发布了其第一轮工作方案计划的大 部分评估报告, 包括《传粉者、传粉和粮食生产评 估》(IPBES, 2016a)、《生物多样性和生态系统服务
情景和模型方法评估》(IPBES, 2016b)、《土地退化 和恢复评估》(IPBES, 2018a), 非洲(IPBES, 2018b)、 美洲(IPBES，2018c)、亚洲-太平洋(IPBES，2018d) 和欧洲-中亚(IPBES, 2018e) 4 个区域尺度的评估报 告以及《生物多样性和生态系统服务全球评估》的 决策者摘要(IPBES, 2019)。“野生物种的可持续利用 和生物多样性”和“生态系统服务多元价值概念化” 这2项评估已完成第一轮外部审查, “外来入侵物种” 评估已经启动。此外，为了第一轮工作方案的顺利 开展, IPBES还成立了三个特别工作组(task force) (即能力建设、土著和地方知识及知识数据工作组) 及若干技术支持机构(technical support unit)。

通过开展第一轮工作方案, IPBES逐渐确立了 其在生物多样性和生态系统服务领域的重要科学 地位。一系列IPBES评估报告的发布, 为了解和掌 握全球生物多样性和生态系统服务相关现状、变化 趋势及其驱动因素提供了科学依据，也为决策者提 供了政策支持工具和方法，成功创造了一批新概念 和新方法，如自然及自然对人类贡献、土著和地方 知识持有者合作模式等(Díaz et al, 2015; Pascual et al，2017); 同时也促成了一些新的科学工作的开展, 如生物多样性和生态系统服务情景设定和模型的 研究与开发(Pascual et al, 2017)。通过编写IPBES评 估报告, 所有参与者都在这一大规模的联合学术研 究中受益匪浅，增进了国际学术合作和交流，也为 成功制定IPBES第二轮工作方案奠定了良好的基 础。此外, IPBES在第一轮工作方案期间通过开展一 系列能力建设活动, 为提高发展中国家研究人员的 专业水平和数据获取能力做出了重要贡献。

\section{2 存在问题}

毫无疑问, IPBES第一轮工作方案取得了丰硕 的成果，但在开展过程中也发现了一些关键问题。

(1)经费来源无法保障, 使平台一度面临财政 赤字问题。由于IPBES资金机制是各成员国自愿捐 款，资金主要依赖于发达国家的捐助，经费来源不 稳定, 导致话语权长期掌握在捐款较多的发达国家 手中。

(2)四项基本职能发展不均衡。IPBES开展评估 这一职能成效显著，但其他三项职能，尤其是“政 策支持”职能未能达到预期目标。由于IPBES对开展 评估职能在成果产出、人力资源和预算方面的投入 均优先于其他三项职能; 且IPBES将评估报告作为 
影响政策的最终成果, 有时甚至规避提供政策选择 这一棘手却非常重要的问题, 认为知识只要“存在”, 就会产生影响，政策支持职能则主要通过开发广泛 的在线政策支持工具清单来实现。然而, 评估结果 能否产生长期、持久的变革性影响, 很大程度上取 决于其他三项职能能否成功发挥作用。

(3)缺乏灵活性。灵活性缺失主要体现在两方面: 一是制定方案时未考虑人力、经费等多种因素, 过 早确定了评估专题和方法, 各缔约方和利益做关方 准备不足, 导致3项评估延迟开展; 二是在制定方 案的初始阶段便明确了方案目标和评估专题等要 点, 且平台与各成员国间缺少互动和交流, 导致尽 管已发现四项基本职能协同增效不理想, 但因方案 缺乏灵活性而无法调整。

(4)工作方案涉及的评估报告还存在一些共性 问题。比如, 有些用于评估的数据较为陈旧, 报告 评估周期一般为3-4年, 所用数据甚至有 10 年前的, 降低了评估结果的可参考性和对政策的现实指导 意义; 由于考虑区域等各方面平衡问题，导致入选 为评估报告作者的专家水平参差不齐; 由于评估作 者背景文化差异、语言限制等，导致数据和信息等 评估证据的置信度低; 未能充分利用四个联合国伙 伴组织(联合国粮食及农业组织、联合国开发计划 署、联合国环境署和联合国教育、科学及文化组织) 及其他多边环境协定的潜在价值, 利益做关方参与 工作的程度有限。尽管如此, IPBES已经在有限的资 源和条件下尽量做到了科学、合理与公平，同时识 别了现有知识的空缺。

\section{IPBES第二轮工作方案(2019-2030}

第一轮工作方案存在的问题为完善第二轮工 作方案提供了经验教训。IPBES第二轮工作方案采 用了灵活的“滚动式”方案，即在方案执行的过程中, 根据需要或新出现的问题, 灵活地确定方案要点和 评估专题, 而不是在方案的起始阶段就明确未来十 年将要开展的工作。此外, 为了加强IPBES四大职 能间的协同增效作用, IPBES将以更加综合的方式 构建第二轮工作方案, 对于IPBES选定的任何评估 专题, 都将有完整的评估内容、能力建设、政策建 议和知识生成四个方面的配套计划和内容, 以促进 IPBES四项基本职能均衡发挥。在第一轮工作方案 的基础上, 第二轮工作方案将进一步帮助人类了解
人与自然之间的关系, 特别是自然对良好生活品质 的贡献。此外, 为了保障第二轮工作方案的顺利开 展，IPBES开始寻求更广的资金来源，如与私营部 门建立合作伙伴关系等。

\section{1 制定过程及各方关注焦点}

2018年，为保障IPBES未来工作方案的包容、 公正、透明，根据IPBES第六次全体会议的决定 ${ }^{(1)}$, 多学科专家组和主席团在秘书处的支持下，分别就 “IPBES第二轮工作方案草案”的编制步骤和范围、 优先主题和具体内容(包括总体结构、目标、可交付 成果和体制安排)等方面，两次征求各成员国和利 益敒关方意见，编制形成“IPBES第二轮工作方案草 案(2019-2030)”(文件IPBES/7/6) ${ }^{2}$ 供第七次全体会 议审议。

在IPBES第七次全体会议上，各方基本认同方 案的优先评估主题, 但由于利益诉求及侧重点不同, 各方在评估专题的时间安排及预算、与联合国政府 间气候变化专门委员会(IPCC)的合作形式和成果发 布方式及是否开展生态连通性评估等问题上存在 分歧。

关于时间安排，美国、南非及日本等国代表对 资金和人力资源是否充裕表示担忧，认为同时开展 多项评估将造成较大的资金及人力资源压力，可能 影响IPBES的正常运作，建议推迟一年启动商业对 生物多样性和自然贡献的影响和依赖的评估(以下 简称“商业评估”。欧盟、荷兰等对此表示反对，同 时建议提前一年开展“生物多样性丧失的根本原因 和实现2050年生物多样性愿景所需转型变革的决 定因素及备选方案”专题评估(以下简称“转型变革 评估”)。全会最终决定，商业评估启动由2019年推 迟至2020年, 转型变革评估则由2020年提前至2019 年启动。

关于与IPCC合作编写《生物多样性和气候变 化》技术文件，美国提出应该明确合作形式及成果 发布方式，主张由IPBES独立发布该项技术文件， IPCC以协助方的角色参与相关工作。欧盟、土耳其、 英国等表示支持弱化IPCC在此次合作中发挥的作 用。此外，由于合作双方在一些具体细节上还没有

\footnotetext{
(1) IPBES 6/2: Development of a Draft Strategic Framework up to 2030 and Elements of the Rolling Work Programme of the Platform. https://www.ipbes.net/system/tdf/decisions_ipbes_6_2_en_0.pdf?file=1\&typ e=node\&id=29492

(2) IPBES/7/6: Future Work Programme of the Platform. https://ipbes. net/event/ipbes-7-plenary.
} 
达成一致, 所以该项工作的启动在一定程度上也受 到了影响。

关于是否开展生态连通性评估 ${ }^{\circledR}$, 《联合国防治 荒漠化公约》(UNCCD)代表首次提出增设生态连通 性评估专题, 加纳、沙特阿拉伯等表示支持, 认为 生态连通性这一专题对整合2030年可持续发展目 标具有重要意义, 但美国、英国和瑞典等指出增加 新的评估将加大资金及人力资源压力，建议推迟讨 论是否增设生态连通性评估问题; UNCCD、西班 牙、摩洛哥、加纳、沙特阿拉伯、玻利维亚和《湿 地公约》最终同意将问题推迟至IPBES第九次全体 会议上讨论。

\section{2 优先主题及主要内容}

第二轮工作方案确定的三个优先主题包括: (1) 生物多样性在实现2030年可持续发展议程方面的 重要性; (2)生物多样性丧失的根本原因和实现2050 年生物多样性愿景所需转型变革的决定因素及备 选方案; (3)商业对生物多样性和自然贡献的影响和 依赖。

在优先主题的基础上，第二轮工作方案设置了 六项目标, 包括开展评估、能力建设、加强知识基 础、政策支持、交流和外联以及提升效率，这些目 标将由一系列预期成果支撑, 并于 5 年内完成已确
定的专题评估工作。第二轮工作方案是一个开放性 方案，IPBES将不定期调整评估专题，因此目前方 案实施内容仅安排至2025年，2026-2030年的工作 计划将在IPBES第十次全体会议上进行讨论。

根据各项评估的时间安排(图1), 2019年开展 “生物多样性、水、粮食和健康之间的相互联系”专 题评估(以下简称“联系评估”)、“转型变革评估”和 “生物多样性与气候变化之间的关联性”技术报告 (以下简称“气候变化报告”); 2020年开展“商业评 估”。第一轮工作方案中未完成的3项评估将延迟至 第二轮工作方案中继续开展, 其中, 《野生物种可 持续利用》专题评估报告和《生物多样性和生态系 统服务多元价值概念化》评估报告将在IPBES第九 次全体会议上进行审议; 《外来入侵物种》评估报 告将在IPBES第十次全体会议上进行审议。

此外, 为有效支撑评估, 设立了五个特别工作 组, 实现除评估职能外的五个职能。

\section{3 对中国参与IPBES工作的建议}

IPBES第二轮工作方案的时间与《2030年可持 续发展议程》及其目标、《生物多样性公约》“2020 后框架”，以及其他环境协定阶段性目标的时间相 重合，且应这些相关公约和环境协定的要求，

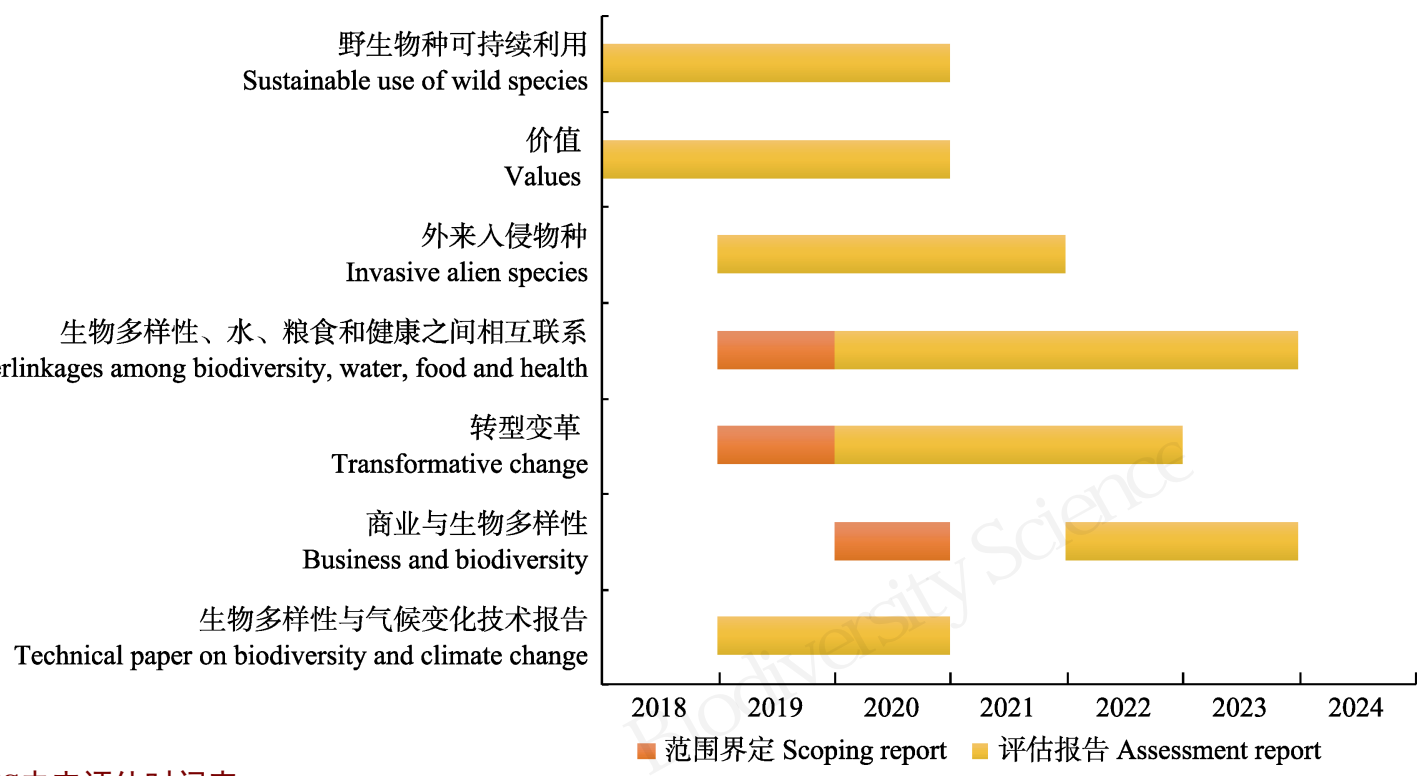

图1 IPBES未来评估时间表

生物多样性、水、粮食和健康之间相互联系 Interlinkages among biodiversity, water, food and health

Fig. 1 The timeline of IPBES assessments in future

(1) 连通性评估: 评估景观对生态网络的基本组成部分(例如土壤、水、生物群)间的流动和联系的促进作用, 提供政策和实践建议。评估主要包括: 景 观连通性、生态连通性、栖息地连通性、进化过程连通性、社会生态连通性以及多尺度生态系统管理与连通性。 
IPBES 对其目标实现情况和成果进行评估, 将为其 谈判磋商提供科学的证据(Bridgewater, 2019)。《生 物多样性公约》第 15 次缔约方大会(COP15)将于 2021 年在中国举办, 其主题为: “生态文明: 共建地 球生命共同体”, 会议的重要议题之一将是围绕 “2020 后框架”进行磋商, 以期制定出至 2030 年的 全球生物多样性保护目标及行动计划, 接替 《2011-2020 生物多样性战略计划》及其爱知生物 多样性目标。“2020 后框架”关系到我国未来生物多 样性保护工作的重点发展方向, 框架能否达成一致, 直接关系到我国能否在保障国家利益的基础上顺 利举办 COP15 (刘文静等, 2018)。由此可见, IPBES 对全球生物多样性与生态系统服务的可持续利用 与保护、经济社会可持续发展以及人类福祉等产生 的影响越发深远。基于此, 对中国参与 IPBES 工作 提出以下几点建议:

(1)加强多部门多学科协作。IPBES 与《生物多 样性公约》、《联合国防治荒漠化公约》和《保护世 界文化和自然遗产公约》等 7 个国际环境公约相关, 其评估领域涉及多个行业和部门，评估结果将以 “评估报告-政府关注一限制性措施”的模式对全球相 关政策产生深远影响(贾翔宇等, 2018)。第二轮工作 方案中计划开展的联系评估、转型变革评估和商业 评估更是涉及到社会、经济和文化多个领域, 需要 农业、渔业、林业和商务等多个部门的支持。因此, 建立健全相关部门的生物多样性管理机构和跨部 门协调机制, 加强多部门多学科协作, 充分利用各 部门的资源和能力, 吸纳更多学科专家参与 IPBES 的评估工作尤为重要。通过建设和完善各领域专家 库、数据库以及全国生物多样性信息网络系统, 协 调使用各部门现有数据库, 建立信息交换和共享机 制，可大大提高各部门工作效率及资源利用率。

(2)加强国际合作与交流。近年来，中国把生态 文明建设作为最优先的战略之一, 充分认识到了尊 重自然、顺应自然、保护自然的重要性，采取了一 系列走在世界前列的政策。“构建人类命运共同体, 实现共赢共享”的中国方案、“绿水青山就是金山银 山”的理念、“生态保护红线”的划定及“污染防治攻 坚战”等一系列的“中国智慧”充分展示了中国推动 全球可持续发展进程, 保护生物多样性的决心。然 而这些理念在国际平台上的宣传还有很大空间。积 极参与 IPBES, 与各国及相关国际组织建立并加强
生物多样性领域的合作与交流，将生态文明建设的 “中国智慧”通过高层对话、国际论坛、交流研讨会 等形式向国际社会宣传推广，树立我国的大国形象， 有助于提升我国在生物多样性领域相关谈判中的 主动权和话语权。与此同时，加强国际协作与交流 也将大大促进我国学习国际上的成功案例及政策 工具和方法。

(3)提升国内研究能力。《生物多样性公约》等 环境公约针对 IPBES 第二轮工作方案提出了建议, 提出开展相关专题评估以满足 “2020 后框架”和 《2030 年可持续发展议程》涉及的有关生物多样性 和生态系统服务的评估需求。各成员国在参与 IPBES 评估的同时，也是在向全球展现各自在相关 领域的科研实力。在第一轮工作方案实施进程中发 现, 我国在相关领域缺乏本土的研究基础, 研究成 果不具国际竞争力, 研究能力(如生物多样性和生 态系统服务情景和模型)尚待提高(潘玉雪等, 2018), 甚至有些评估专题(如传粉者、传粉和粮食生产)处 于本底不清状态(田瑜等, 2016)。鉴 IPBES 的相关 评估正受到越来越多政府和国际组织的关注，且能 够影响相关公约的谈判进程，建议在国内积极开展 第二轮工作方案相关专题评估, 针对研究空缺发展 学科建设和基础研究，加大对相关专题的资金投入, 提升国内研究能力。同时要加快建设国内生物多样 性综合信息平台，进一步做好数据的存储、管理和 应用, 为今后的评估工作提供数据基础。

(4)加强能力建设, 扩大宣传。能力建设是 IPBES 的四项基本职能之一, IPBES 全体会议设立 专门的能力建设特别工作组, 并启动了能力建设滚 动计划和奖学金计划。IPBES 将为第二轮工作方案 的每项评估专题配备相应的能力建设活动。其中, IPBES 青年学者项目是 IPBES 能力建设工作组的突 出成果之一。该项目在每次篎选评估报告作者时启 动, 在全球选拔优秀的青年学者为 IPBES 评估报告 的撰写提供协助, IPBES 将为其安排一名导师, 并 邀请其参加评估报告的作者会议和能力建设研讨 会, 为青年学者提供与国际杰出专家交流学习的机 会。《生物多样性公约》对 IPBES 的能力建设工作 给予了充分肯定。充分参与相关能力建设活动, 吸 收借鉴 IPBES 能力建设工作经验对国内工作有极 大的指导意义, 包括考虑设立奖学金计划和青年学 者项目, 培养和推荐一批优秀的青年学者参与 
IPBES 或国内的相关工作，并通过培训、对话会、 研讨会和媒体宣传等形式提升能力，同时扩大生物 多样性的社会影响力, 提升公众认知和参与。针对 即将开展的商业评估，应加强对企业管理人员的宣 传和培训，提升其保护意识，鼓励企业参与到生物 多样性保护工作中来。

\section{参考文献}

Bridgewater P, Loyau A, Schmeller DS (2019) The Seventh Plenary of the Intergovernmental Platform for Biodiversity and Ecosystem Services (IPBES-7): A global assessment and a reshaping of IPBES. Biodiversity and Conservation, 28, 2457-2461.

Díaz S, Demissew S, Carabias J, Joly C, Lonsdale M, Ash N, Larigauderie A, Adhikari JR, Arico S, Báldi A, Bartuska A, Baste IA, Bilgin A, Brondizio E, Chan KMA, Figueroa VE, Duraiappah A, Fischer M, Hill R, Koetz T, Leadley P, Lyver P, Mace GM, Martin-Lopez B, Okumura M, Pacheco D, Pascual U, Pérez ES, Reyers B, Roth E, Saito O, Scholes RJ, Sharma N, Tallis H, Thaman R, Watson R, Yahara T, Hamid ZA, Akosim C, Al-Hafedh Y, Allahverdiyev R, Amankwah E, Asah ST, Asfaw Z, Bartus G, Brooks LA, Caillaux J, Dalle G, Darnaedi D, Driver A, Erpul G, Escobar-Eyzaguirre P, Failler P, Fouda AMM, Fu BJ, Gundimeda H, Hashimoto S, Homer F, Lavorel S, Lichtenstein G, Mala WA, Mandivenyi W, Matczak P, Mbizvo C, Mehrdadi M, Metzger JP, Mikissa JB, Moller H, Mooney HA, Mumby P, Nagendra H, Nesshover C, Oteng-Yeboah AA, Pataki G, Roué M, Rubis J, Schultz M, Smith P, Sumaila R, Takeuchi K, Thomas S, Verma M, Youn YC, Zlatanova D (2015) The IPBES Conceptual Framework-Connecting nature and people. Current Opinion in Environmental Sustainability, 14, 1-16.

IPBES (2016a) The Assessment Report of the Intergovernmental Science-Policy Platform on Biodiversity and Ecosystem Services on Pollinators, Pollination and Food Production. IPBES, Bonn, Germany. https://www.ipbes.net/ assessment-reports. (accessed on 2020-10-17)

IPBES (2016b) The Methodological Assessment Report on Scenarios and Models of Biodiversity and Ecosystem Services. IPBES, Bonn, Germany. https:/www.ipbes.net/ assessment-reports. (accessed on 2020-10-17)

IPBES (2018a) The assessment report on Land Degradation and Restoration. IPBES, Bonn, Germany. https://www.ipbes .net/assessment-reports. (accessed on 2020-10-17)

IPBES (2018b) The IPBES Regional Assessment Report on Biodiversity and Ecosystem Services for Africa. IPBES, Bonn, Germany. https://www.ipbes.net/assessment-reports. (accessed on 2020-10-17)

IPBES (2018c) The IPBES Regional Assessment Report on Biodiversity and Ecosystem Services for the Americas. IPBES, Bonn, Germany. https://www.ipbes.net/assessmentreports. (accessed on 2020-10-17)

IPBES (2018d) The IPBES Regional Assessment Report on Biodiversity and Ecosystem Services for Asia and the Pacific. IPBES, Bonn, Germany. https://www.ipbes.net/ assessment-reports. (accessed on 2020-10-17)

IPBES (2018e) The IPBES Regional Assessment Report on Biodiversity and Ecosystem Services for Europe and Central Asia. IPBES, Bonn, Germany. https://www.ipbes.net/assess ment-reports. (accessed on 2020-10-17)

IPBES (2019) Global Assessment on Biodiversity and Ecosystem Services. IPBES, Bonn, Germany. https:/www. ipbes.net/event/ipbes-7-plenary. (accessed on 2020-10-17)

Jia XY, Bai B, Zhang JQ, Huang Y (2018) The effects of IPBES deliverables on global biodiversity conservation strategy-An analysis based on the U. S. pollinator protection policy. Biodiversity Science, 26, 527-534. (in Chinese with English abstract) [贾翔宇, 白彬, 张洁清, 黄 艺 (2018) IPBES评估报告对全球生物多样性保护的影响 -以美国传粉者保护政策为例. 生物多样性, 26, 527-534.]

Liu WJ, Xu J, Geng YJ, Tian Y, Yin SL (2018) Progress in negotiations on the Post-2020 Global Biodiversity Framework and suggestions for Chinese government. Biodiversity Science, 26, 1358-1364. (in Chinese with English abstract) [刘文静, 徐靖, 耿宜佳, 田瑜, 银森录 (2018) “2020年后全球生物多样性框架”的谈判进展及对 我国的建议. 生物多样性, 26, 1358-1364.]

Pan YX, Tian Y, Xu J, Zhang BY, Li JS (2018) Methodological assessment on scenarios and models of biodiversity and ecosystem services and its impact on China within the IPBES framework. Biodiversity Science, 26, 89-95. (in Chinese with English abstract) [潘玉雪, 田瑜, 徐靖, 张博 雅, 李俊生 (2018) IPBES框架下生物多样性和生态系统 服务情景和模型方法评估及对我国的影响. 生物多样性, 26, 89-95.]

Pascual U, Balvanera P, Díaz S, Pataki G, Roth E, Stenseke M, Watson RT, Başak DE, Islar M, Kelemen E, Maris V, Quaas M, Subramanian SM, Wittmer $\mathrm{H}$, Adlan A, Ahn SE, Al-Hafedh YS, Amankwah E, Asah ST, Berry P, Bilgin A, Breslow SJ, Bullock C, Cáceres D, Daly-Hassen H, Figueroa E, Golden CD, Gómez-Baggethun E, González-Jiménez D, Houdet J, Keune H, Kumar R, Ma KP, May PH, Mead A, O’Farrell P, Pandit R, Pengue W, Pichis-Madruga R, Popa F, Preston S, Pacheco-Balanza D, Saarikoski H, Strassburg BB, van den Belt M, Verma M, Wickson F, Yagi N (2017) Valuing nature's contributions to people: The IPBES approach. Current Opinion in Environmental Sustainability, 26/27, 7-16.

Tian Y, Lan CZ, Xu J, Li XS, Li JS (2016) Assessment of pollination and China's implementation strategies within the IPBES framework. Biodiversity Science, 24, 1084-1090. (in Chinese with English abstract) [田瑜, 兰存子, 徐靖, 李秀 山, 李俊生 (2016) IPBES框架下的全球传粉评估及我国 对策. 生物多样性, 24, 1084-1090.]

Tian Y, Li JS, Lan CZ, Li XS (2015) Interpretation of the work programme of Intergovernmental Science-Policy Platform on Biodiversity and Ecosystem Services for the period 2014-2018. Biodiversity Science, 23, 543-549. (in Chinese with English abstract) [田瑜, 李俊生, 兰存子, 李秀山 (2015) 生物多样性和生态系统服务政府间科学-政策平 台2014-2018年工作方案解析. 生物多样性, 23, 543-549.]

(责任编委: 薛达元 责任编辑: 时意专) 